\section{Introduction: Addressing Challenges in Multiple Myeloma Management in an Era of New Therapeutics}

Hematologists/oncologists and other physicians can expect to encounter an increasing number of patients with multiple myeloma (MM) in the coming years. Between 1997 and 2006, the incidence rate of myeloma declined in the United States, but the burden (number of incident cases) increased. ${ }^{1}$ An analysis of population-based cancer registries in 9 countries detected modest increases in the incidence of MM in most between 1973 and 1992, with further increases projected by 2007. ${ }^{2}$ MM remains the second most common hematologic malignancy in the United States after nonHodgkin's lymphoma. ${ }^{3}$

Historically, MM has been a difficult and frustrating disease for patients and physicians. Since the reintroduction of thalidomide and development of lenalidomide and bortezomib, outcomes have improved considerably, particularly when these newer agents are combined with conventional chemotherapeutic agents or each other. Using the first-line regimens common in 1983, typical 2 -year survival rates were $48 \%$ to $66 \%$. In trials of triple combination therapy reported in 2008, the 2-year survival rates ranged from $83 \%$ to $90 \% .{ }^{4}$ In the United States, the 5-year survival rate improved from 26\% in 1975 to 1977 to $34 \%$ in 1996 to 2003, a statistically significant difference. ${ }^{5}$

Obviously, it is vitally important for today's physicians to understand the newer agents, but this is a challenge because the drugs have multiple functions (e.g., antiangiogenesis, immunomodulation, proteasome inhibition), have different toxicity profiles, and can be used in myriad combinations and sequences. Hematologists/ oncologists have indicated a need to know more about choosing first-, second-, and third-line therapies; side effects of the new agents; the role and timing of stem cell transplantation in the era of new therapies; guidelines for maintenance therapy; and what new therapeutic combinations will be major factors in treating myeloma in the coming years. ${ }^{6-8}$

This supplement is part of a 3-supplement series planned by myeloma experts via a roundtable discussion held April 24, 2009, in Philadelphia. Our charge was to develop outlines of 10 articles about myeloma therapy that would convey the information currently of most importance to community oncologists. The first 2 of the 4 articles in this supplement address risk reduction and management of the most common complications of myeloma therapy. Dr. Paul Richardson and colleagues focus on how thalidomide- and bortezomib-induced peripheral neuropathy usually can be effectively managed with dose reduction, schedule modification, optimized agent sequencing, or a combination of nonpharmacologic and pharmacologic therapy for symptom relief. They also discuss asthenia (fatigue), an important topic that is too infrequently addressed in myeloma reviews. The companion article, by Drs. Ruben Niesvizky and Ashraf Badros, explains the measures that oncologists should take to protect patients from a life-threatening complication, venous thromboembolism, the risk of which is elevated with thalidomide and lenalidomide. That article also reviews the latest data on osteonecrosis of the jaw, renal complications, and anemia.

Dr. Badros has contributed 2 additional articles to this supplement: one on maintenance therapy and the other on defining and treating high-risk myeloma. He explains that only recently, after 3 decades of investigation, have certain maintenance regimens shown promise in myeloma. In particular, several studies suggest that thalidomide, alone or with a corticosteroid, prolongs overall survival after autologous stem cell transplantation (ASCT). However, other studies have failed to support this
Sundar Jagannath, MD

From St. Vincent's

Comprehensive Cancer Center, New York, New York.

Correspondence: Sundar Jagannath, MD, St. Vincent's Comprehensive Cancer Center, 325 West 17th Street, New York, NY 10011. E-mail: sjagannath@ aptiumoncology.com 
conclusion, and several important questions remain. Bortezomib- and lenalidomidebased maintenance therapies are being evaluated in ongoing randomized trials.

In the final article, Dr. Badros notes that advances in genetic analysis have allowed better estimates of prognosis for patients with myeloma. An effective means of risk stratification is required so that patients at high risk may be offered more aggressive treatment or be entered into clinical trials of investigational agents as firstline therapy. Dr. Badros discusses evolving risk stratification systems and the potential of the newer agents to improve outcomes in patients with newly diagnosed high-risk myeloma.

The first supplement in this series was published in Community Oncology. In the opening article on treatment goals, I review the indications for treating MM, help set goals for first-line treatment and treatment of relapsed/refractory disease, and suggest how to reconcile different schools of thought about treating MM.

In the second article in the Community Oncology supplement, Dr. Sergio Giralt reviews recent data on the use of novel therapies in the transplant setting and discusses why ASCT remains a standard of care in myeloma.

In the final article of the supplement, Dr. Donald Siegel and Elizabeth Bilotti discuss the investigational antimyeloma agents that are farthest along in clinical development.

The third supplement in this series, entitled "Essentials for Tailoring Multiple Myeloma Therapy," will be published later this year by Oncology. It compares efficacy and safety data for the various therapies recommended for patients with newly diagnosed myeloma and those with relapsed/refractory disease. In it, Dr. Edward Stadtmauer provides guidance for choosing among the NCCN recommended regimens, with particular attention to patients who have cytogenetic abnormalities, renal disease, or other negative prognostic indicators. Dr. Niesvizky and colleagues discuss front-line treatment of patients with myeloma who cannot or choose not to undergo ASCT. Finally, Dr. Richardson and colleagues close the series by reviewing the treatment of relapsed/refractory disease.

An estimated $90 \%$ of oncology care in the United States is performed outside of academic medical centers, ${ }^{9}$ and data suggest that uptake of new evidence can be as fast in the community as at academic medical centers. ${ }^{10-13}$ The hope is that the information in these supplements will help community oncologists understand the latest standards for myeloma care and feel more confident about participating in this exciting, fast-changing field.

\section{References}

1. Horner MJ, Ries LA, Krapcho M, et al, eds. SEER Cancer Statistics Review, 1975-2006, National Cancer Institute. Bethesda, MD. Based on November 2008 SEER data submission, posted to the SEER Web site 2009. Available at: http://seer.cancer.gov/csr/1975_2006. Accessed May 8, 2009.

2. Bray I, Brennan P, Boffetta P. Recent trends and future projections of lymphoid neoplasms—a Bayesian age-period-cohort analysis. Cancer Causes Control 2001;12:813-820.

3. American Cancer Society. Cancer Facts \& Figures 2009. Available at: www.cancer.org/downloads/ STT/500809web.pdf. Accessed May 20, 2009.

4. Durie BG. Myeloma therapy: 25 years forward-immune modulation then and now. J Clin Oncol 2008;26:4698-4700.

5. Jemal A, Siegel R, Ward E, et al. Cancer statistics, 2008. CA Cancer J Clin 2008;58:71-96.

6. Panel Intelligence. Multiple myeloma market assessment. A panel discussion among ten hematologists/ oncologists. Cambridge, MA; March 2007. Available at: www.medpanel.com/reports/multiple_ myleoma_therapy_market_assessment. Accessed February 11, 2009. 
Introduction

7. Panel Intelligence. Multiple myeloma: US market assessment. A survey of fifty community-based hematologist/oncologists. Cambridge, MA; March 2007. Available at: www.medpanel.com/reports/ multiple_myeloma_us_market_assesment. Accessed February 11, 2009.

8. Myeloma Euronet International survey on barriers to myeloma diagnosis, treatment, and care 2006. February 2007. Available at: www.myeloma-euronet.org/en/survey/index.php. Accessed May 30, 2008.

9. PR Newswire. "Oncology World Congress survey finds wide gap between urban and rural oncologists in resource and information access" [press release]. September 20, 2004. Available at: www2.prnewswire. $\mathrm{com} / \mathrm{cgi}$-bin/stories.pl?ACCT $=104 \& S T O R Y=/ \mathrm{www} /$ story/09-20-2005/0004111294\&EDATE. Accessed October 5, 2009.

10. Angermund R, Knauf W, Freund M, et al. Role of bortezomib in the treatment of multiple myelomafirst results from a representative multicentre treatment survey in Germany [abstract]. Blood 2005; 106:Abstract 5156.

11. Cheung MC, Loutfy MR, Leitch HA, et al. Physician perceptions and preferences in the treatment of acquired immunodeficiency syndrome related lymphoma [abstract]. Blood 2006;108:Abstract 3331.

12. Tummala MK, Wajahath $M$, Kotlarewsky M, et al. Patterns of care regarding adjuvant hormonal agents and treatment of bone health in postmenopausal women with breast cancer in community and academic centers before and after the availability of ATAC (Adjuvant Tamoxifen, Arimidex or Combination) study [abstract]. J Clin Oncol 2007;25(Suppl 1):Abstract 6621.

13. McKibbin T, Kwan P, Simon J, Koeller J. Rapid adoption of chemotherapy regimens for advanced colorectal cancer (CRC) in the community oncology setting [abstract]. J Clin Oncol 2008;26(Suppl 1):Abstract 6563 . 